\title{
Asymmetries in vertical vorticity and vertical velocity arising during nonlinear homogeneous spindown
}

\author{
J. A. Benthuysen ${ }^{1, a)}$ and L. N. Thomas ${ }^{2, b)}$ \\ 1) MIT/WHOI Joint Program, Woods Hole, MA 02543, \\ $U S A$ c) \\ ${ }^{2)}$ Department of Environmental Earth System Science, Stanford University, \\ Stanford, CA 94305, USA
}

(Dated: 21 May 2012)

During the spindown of a uni-directional, geostrophic current in a homogeneous fluid, asymmetry arises in the vertical velocity and vertical relative vorticity fields. A closed-form, time-dependent solution valid to order Rossby number is derived to explore these asymmetries. Momentum advection in the interior and the Ekman layer leads to competing tendencies in the vertical vorticity's evolution. In the interior, momentum advection hastens spindown in cyclonic regions. In the Ekman layer, momentum advection weakens Ekman pumping over Ekman suction and thus tends to slow the spindown of cyclonic vorticity. It is shown that the former effect dominates, and hence cyclonic vorticity decays faster than anticyclonic vorticity.

\footnotetext{
a) Electronic mail: jessica.benthuysen@csiro.au

b)Electronic mail: leift@stanford.edu

c) Also at CSIRO Marine and Atmospheric Research, Hobart, Australia.
} 
Asymmetries in vertical vorticity and vertical velocity arising during nonlinear homogeneous spindown

\section{INTRODUCTION}

The adjustment of a geostrophically balanced flow over a boundary produces frictionally driven circulations, in which convergences in the Ekman transport eject fluid out of the boundary layer (Ekman pumping) and divergences in the Ekman transport inject fluid into the boundary layer (Ekman suction). The magnitude and lateral structure of Ekman pumping and suction determine the strength and structure of interior secondary circulations that spin down the geostrophic flow. In the linear Ekman balance, the vertical velocity at the top of the frictional boundary layer is dependent on the vertical component of the geostrophic relative vorticity, $\zeta$ (Charney and Eliassen ${ }^{1}$ ). Then, Ekman pumping is symmetric to Ekman suction for a change in the sign of the vertical relative vorticity. The purpose of this work is to examine how nonlinearity breaks this symmetry between Ekman pumping and suction and modifies the geostrophic vertical vorticity field during spindown.

Previous studies have shown that Ekman advection of momentum in a homogeneous fluid over a flat bottom weakens Ekman pumping for cyclonic flows, $\zeta>0$, and strengthens Ekman suction for anticyclonic flows, $\zeta<0$, at $O(\epsilon), \epsilon=U / f L$, where $U$ is the characteristic flow speed, $f$ is the planetary vorticity and $f>0$ is assumed in this work, and $L$ is the characteristic lateral length scale. For flows with lateral shear that is temporally and spatially constant, Benton et $a .^{2}{ }^{2}$ identified that the vertical velocity was stronger for anticyclonic flows than cyclonic flows at $O(\epsilon)$. For steady general shear lines and circular vortices, Hart $^{3}$ analytically solved for higher order corrections to nonlinear Ekman pumping. Horizontal Ekman advection of momentum weakens Ekman pumping and strengthens Ekman suction at $O(\epsilon)^{3}$. Further examination of the steady Ekman layer reveals that the Ekman layer depth is modified from its linear value, $\delta_{e}=\sqrt{2 \nu / f}$, by momentum advection ${ }^{4,5}$, where $\nu$ is the kinematic viscosity. For general shear lines, a formal perturbation expansion demonstrates that vertical advection of momentum causes the Ekman layer to thicken in cyclonic regions and to thin in anticyclonic regions ${ }^{5}$. With the nonlinear Ekman layer depth, the $O(\epsilon)$ correction to Ekman pumping remains unchanged from Hart's ${ }^{3}$ formula ${ }^{5}$. These studies detail the contributions of horizontal and vertical momentum advection in the nonlinear modification of the Ekman layer thickness and Ekman pumping. However, the feedback between nonlinear Ekman pumping and the interior vertical vorticity field is a fundamental aspect of the time-dependent spindown problem. 
Asymmetries in vertical vorticity and vertical velocity arising during nonlinear homogeneous spindown

The nonlinear spindown of a homogeneous, geostrophic current is subject to nonlinear Ekman pumping as well as horizontal momentum advection in the interior. $\mathrm{Hart}^{6}$ derived an $O(\epsilon)$ accurate equation for vertical vorticity, and, for the special case of spatially uniform vertical vorticity, determined an approximate solution for its time-dependent decay. The solution showed that spatially uniform cyclones spin down faster than anticyclones, even though Ekman pumping is weaker than Ekman suction ${ }^{6}$. In the present study, a more general initial vertical vorticity field is used to illustrate how horizontal momentum advection in the interior dominates over nonlinear Ekman pumping.

Other numerical ${ }^{7,8}$ and laboratory ${ }^{8}$ experiments have also indicated that momentum advection in the interior controls the asymmetric spindown of vertical vorticity. Zavala Sansón and van Heijst ${ }^{7}$ compared the homogeneous spindown of cyclonic vortices in a laboratory experiment with a model, in which momentum advection in the interior was included but the nonlinear correction to Ekman pumping was neglected. They justified neglecting the nonlinear correction to Ekman pumping due to good agreement between results from the laboratory and numerical model. Numerical simulations for the spindown of axisymmetric vortices subject to linear Ekman pumping and suction showed a faster decay in cyclonic vortices than anticyclonic vortices. This behavior is consistent with the hypothesis that horizontal momentum advection in the interior dominates over the effects of nonlinear Ekman pumping and suction ${ }^{6-8}$. Thus, the question remains regarding why nonlinear Ekman pumping plays a subdominant role during nonlinear spindown.

This work aims to more generally quantify the relative roles of nonlinear Ekman pumping and horizontal momentum advection during nonlinear spindown. The problem is formulated in Section II for a general, uni-directional flow that is horizontally bounded or periodic. The time-dependent, $O(\epsilon)$ problem is solved in Section III. This solution shows that horizontal momentum advection in the interior controls the asymmetric spindown of cyclonic and anticyclonic vorticity and, in doing so, enhances the asymmetry in Ekman pumping and suction from the magnitude predicted by Hart's formula ${ }^{3}$. In Section IV, the results are compared with past studies and conclusions are presented. 


\section{FORMULATION}

The equations for a homogeneous fluid rotating about the vertical axis at an angular velocity $f / 2$ are

$$
\begin{gathered}
\frac{\partial \boldsymbol{u}}{\partial t}+\boldsymbol{u} \cdot \nabla \boldsymbol{u}+f \hat{k} \times \boldsymbol{u}=-\frac{1}{\rho_{o}} \nabla p-g \hat{k}+\nu \nabla^{2} \boldsymbol{u}, \\
\nabla \cdot \boldsymbol{u}=0,
\end{gathered}
$$

where $\boldsymbol{u}$ is the velocity field, $p$ is pressure, $\rho_{o}$ is the constant density, $g$ is gravitational acceleration, and $\hat{k}$ is the vertical unit vector. The kinematic viscosity is assumed constant. The flow is assumed to have no spatial variations in the $x$-direction. The fluid is unbounded in the $y$-direction and is confined to a constant depth $H$. The initial, geostrophic flow is assumed either horizontally bounded such that $|\boldsymbol{u}| \rightarrow 0$ as $y \rightarrow \pm \infty$ or periodic with zero spatial average.

The flow, $\boldsymbol{u}$, is decomposed into a frictionless interior region and an Ekman layer, with subscripts $i$ and $e$ denoting the respective domains. Time is nondimensionalized as $t^{\prime}=t / \mathcal{T}_{\text {spindown }}$, where $\mathcal{T}_{\text {spindown }}=E^{-1 / 2} f^{-1}$ is the homogeneous spindown timescale and the Ekman number is defined as $E=\left(\delta_{e} / H\right)^{2}$. On this timescale, the magnitude of vertical vorticity, $\zeta=-\partial u / \partial y$, is shown to decay asymmetrically for cyclonic and anticyclonic vorticity. With primes denoting nondimensional quantities, the variables are nondimensionalized in the interior and Ekman boundary layers by the following set of scalings:

$$
\begin{aligned}
& u_{i}=U u_{i}^{\prime}, \quad u_{e}=U u_{e}^{\prime}, \\
& v_{i}=U E^{1 / 2} v_{i}^{\prime}, \quad v_{e}=U v_{e}^{\prime}, \\
& w_{i}=U E^{1 / 2} \Gamma w_{i}^{\prime}, w_{e}=U E^{1 / 2} \Gamma w_{e}^{\prime}, \\
& p_{i}=\rho_{o} f U L p_{i}^{\prime} .
\end{aligned}
$$

The vertical coordinate is nondimensionalized in the Ekman boundary layer as $\eta^{\prime}=z / \delta_{e}$.

In order to filter inertial oscillations, the Rossby number, $\epsilon=U / f L$, is assumed greater than the Ekman number such that $\epsilon>E^{1 / 2}$ and $E \ll 1$. The aspect ratio, $\Gamma=H / L$, is assumed sufficiently small so that the flow is hydrostatic. Within the Ekman layer, vertical variations in the pressure field are negligible. The upper boundary is assumed rigid, where the Froude number, $F=U / \sqrt{g H}$, satisfies $F<\epsilon E^{1 / 4}$. The flow is subject to the following 
Asymmetries in vertical vorticity and vertical velocity arising during nonlinear homogeneous spindown

no-slip and no normal flow boundary conditions:

$$
\begin{aligned}
u=v & =0 \text { at } z=0, \\
w & =0 \text { at } z=0 \text { and } H .
\end{aligned}
$$

The interior equations to $O(\epsilon)$, primes dropped, become

$$
\begin{aligned}
\frac{\partial u_{i}}{\partial t}+\epsilon v_{i} \frac{\partial u_{i}}{\partial y}-v_{i} & =0 \\
u_{i} & =-\frac{\partial p_{i}}{\partial y}, \\
\frac{\partial v_{i}}{\partial y}+\frac{\partial w_{i}}{\partial z} & =0 .
\end{aligned}
$$

The Ekman layer equations to $O(\epsilon)$, where slow variations in the Ekman layer thickness are not explicitly expressed, become

$$
\begin{aligned}
\epsilon v_{e} \frac{\partial\left(u_{e}+u_{i}\right)}{\partial y}+\epsilon\left(w_{e}+w_{i}(z=0)\right) \frac{\partial u_{e}}{\partial \eta}-v_{e} & =\frac{1}{2} \frac{\partial^{2} u_{e}}{\partial \eta^{2}} \\
\epsilon v_{e} \frac{\partial v_{e}}{\partial y}+\epsilon\left(w_{e}+w_{i}(z=0)\right) \frac{\partial v_{e}}{\partial \eta}+u_{e} & =\frac{1}{2} \frac{\partial^{2} v_{e}}{\partial \eta^{2}}, \\
\frac{\partial v_{e}}{\partial y}+\frac{\partial w_{e}}{\partial \eta} & =0 .
\end{aligned}
$$

The interior vertical velocity is evaluated at $z=0$ because vertical variations of the interior flow within the Ekman layer are $O\left(E^{1 / 2}\right)$. The nondimensional boundary conditions to $O(\epsilon)$ become

$$
\begin{aligned}
u_{i}(z=0)+u_{e}(\eta=0) & =0, \\
v_{e}(\eta=0) & =0, \\
w_{i}(z=0)+w_{e}(\eta=0) & =0, \\
w_{i}(z=1) & =0, \\
u_{e}, v_{e}, w_{e} \rightarrow 0 & \text { as } \eta \rightarrow \infty .
\end{aligned}
$$

The flow variables are expanded in terms of the Rossby number as $\boldsymbol{u}=\boldsymbol{u}^{(0)}+\epsilon \boldsymbol{u}^{(1)}$. The Ekman pumping solution ${ }^{3,5}$ to $O(\epsilon)$ is modified to include time-dependent feedback with the $O(\epsilon)$ geostrophic flow, where

$$
\begin{aligned}
w_{i}(z=0)= & -\frac{1}{2} \frac{\partial u_{i}^{(0)}}{\partial y} \\
& -\frac{7 \epsilon}{40}\left(u_{i}^{(0)} \frac{\partial^{2} u_{i}^{(0)}}{\partial y^{2}}+\left(\frac{\partial u_{i}^{(0)}}{\partial y}\right)^{2}\right)-\frac{\epsilon}{2} \frac{\partial u_{i}^{(1)}}{\partial y} .
\end{aligned}
$$


Asymmetries in vertical vorticity and vertical velocity arising during nonlinear homogeneous spindown

This expression for Ekman pumping includes the first term, which is symmetric in magnitude when the vertical relative vorticity changes sign, the second term, which is Hart's ${ }^{3}$ nonlinear correction to Ekman pumping due to Ekman advection of momentum, and the third term, which represents feedback with the time-dependent, $O(\epsilon)$ vertical relative vorticity.

\section{RESULTS}

\section{A. $O(1)$ solution}

At leading order, the geostrophic flow spins down by the $O(1)$ secondary circulation, where

$$
\frac{\partial u_{i}^{(0)}}{\partial t}-v_{i}^{(0)}=0
$$

The solution to the flow is determined by vertically integrating the interior continuity equation and applying the Ekman pumping condition. The resulting expression

$$
-\frac{\partial v_{i}^{(0)}}{\partial y}=-\frac{1}{2} \frac{\partial u_{i}^{(0)}}{\partial y}
$$

is horizontally integrated from $y_{0}$ to $y$, under the constraint that $u\left(y_{0}\right)=v\left(y_{0}\right)=0$. This constraint is applicable for flows that are horizontally bounded, in which $|\boldsymbol{u}| \rightarrow 0$ as $y \rightarrow$ $\pm \infty$, or periodic with zero horizontal average. Then, the secondary circulation is given by

$$
\begin{aligned}
v_{i}^{(0)} & =-\frac{1}{2} u_{i}^{(0)} \\
w_{i}^{(0)} & =-\frac{1}{2} \frac{\partial u_{i}^{(0)}}{\partial y}(1-z),
\end{aligned}
$$

which is nonzero at initial time due to its spinup on the inertial timescale. By integrating (17) in time, the leading order geostrophic flow decays as

$$
u_{i}^{(0)}=u_{i}^{(0)}(t=0, y) \mathrm{e}^{-t / 2} .
$$

\section{B. $O(\epsilon)$ solution}

Next, the $O(\epsilon)$ interior flow is solved to show that lateral momentum advection in the interior causes cyclones to spin down faster than anticyclones and enhances the asymmetry 
in Ekman pumping and suction from Hart's ${ }^{3}$ nonlinear correction. The $O(\epsilon)$ equations for the interior flow field are

$$
\begin{aligned}
\frac{\partial u_{i}^{(1)}}{\partial t}+v_{i}^{(0)} \frac{\partial u_{i}^{(0)}}{\partial y}-v_{i}^{(1)} & =0, \\
\frac{\partial v_{i}^{(1)}}{\partial y}+\frac{\partial w_{i}^{(1)}}{\partial z} & =0 .
\end{aligned}
$$

The continuity equation, (23), is vertically integrated, subject to the Ekman pumping condition, (16), and then horizontally integrated from $y_{0}$ to $y$ under the assumption $u_{i}^{(0)}\left(y_{0}\right)=$ $v_{i}^{(0)}\left(y_{0}\right)=0$. The resulting secondary circulation is given by

$$
\begin{aligned}
v_{i}^{(1)} & =-\frac{7}{40} u_{i}^{(0)} \frac{\partial u_{i}^{(0)}}{\partial y}-\frac{1}{2} u_{i}^{(1)}, \\
w_{i}^{(1)} & =\left\{-\frac{7}{40}\left(u_{i}^{(0)} \frac{\partial^{2} u_{i}^{(0)}}{\partial y^{2}}+\left(\frac{\partial u_{i}^{(0)}}{\partial y}\right)^{2}\right)-\frac{1}{2} \frac{\partial u_{i}^{(1)}}{\partial y}\right\} \\
& \times(1-z) .
\end{aligned}
$$

The equation for the $O(\epsilon)$ geostrophic current becomes

$$
\frac{\partial u_{i}^{(1)}}{\partial t}+\frac{1}{2} u_{i}^{(1)}=-\frac{7}{40} u_{i}^{(0)} \frac{\partial u_{i}^{(0)}}{\partial y}+\frac{1}{2} u_{i}^{(0)} \frac{\partial u_{i}^{(0)}}{\partial y} .
$$

The interior geostrophic flow is forced by two opposing components from the $O(1)$ flow. The first term on the right side of (26) is from Hart's ${ }^{3}$ nonlinear Ekman pumping formula, and the second term is from lateral advection of the interior geostrophic flow. Lateral momentum advection in the interior not only dominates the forcing of the $O(\epsilon)$ geostrophic flow but is opposite in sign to the contribution from Hart's ${ }^{3}$ nonlinear Ekman pumping formula. Thus, Hart's ${ }^{3}$ nonlinear Ekman pumping alone predicts a change to the $O(\epsilon)$ geostrophic flow that is of opposite sign than if lateral momentum advection is taken into account. Furthermore, forcing by only lateral momentum advection overestimates the change to the $O(\epsilon)$ geostrophic current. As the $O(1)$ forcing decays in time, the feedback term, which is the second term on the left side of (26), dominates and spins down the $O(\epsilon)$ geostrophic flow.

The nonlinear modification of the geostrophic current can also be represented in terms of vertical relative vorticity. From $(22)$, the $O(\epsilon)$ vertical relative vorticity evolves as

$$
\frac{\partial \zeta_{i}^{(1)}}{\partial t}+\underbrace{v_{i}^{(0)} \frac{\partial \zeta_{i}^{(0)}}{\partial y}}_{\mathrm{I}}=\underbrace{\frac{\partial w_{i}^{(1)}}{\partial z}}_{\mathrm{II}}+\underbrace{\zeta_{i}^{(0)} \frac{\partial w_{i}^{(0)}}{\partial z}}_{\mathrm{III}} .
$$




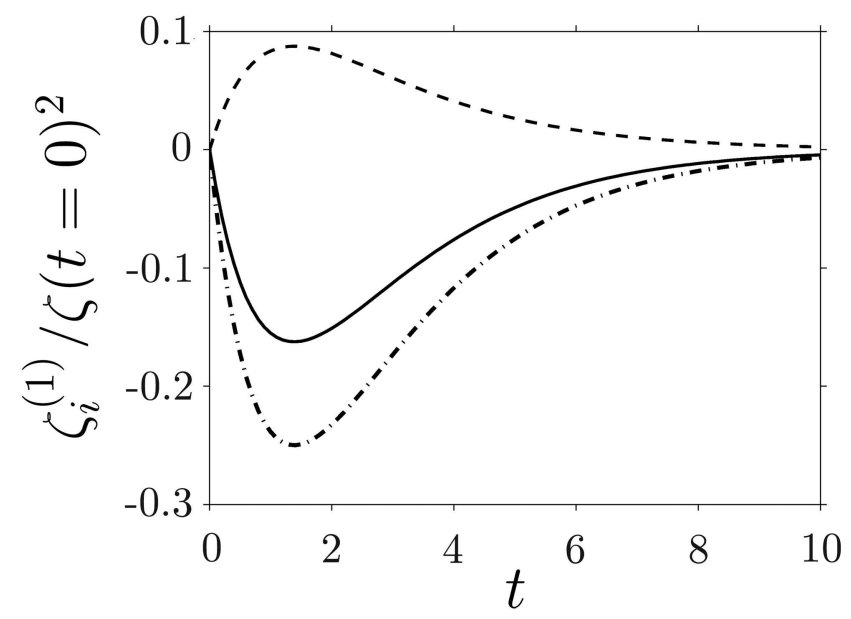

FIG. 1. The $O(\epsilon)$ vertical relative vorticity, which is scaled with the square of the initial vertical relative vorticity, is shown on the cyclonic or anticyclonic axis. The total correction (solid line) has contributions from momentum advection in the interior (dot-dashed line) as well as feedback with the $O(\epsilon)$ Ekman pumping, which includes modification by Ekman advection of momentum (dashed line).

Momentum advection drives lateral advection of vorticity (term I in (27)) and affects the $O(\epsilon)$ contribution to stretching and squeezing of vortex tubes via term III in (27). The vertical velocity generated by nonlinear Ekman pumping and suction also modifies the $O(\epsilon)$ contribution to stretching and squeezing of vortex tubes through term II in (27). Figure 1 compares the contributions to $\zeta_{i}^{(1)}$ from terms II and III evaluated at the cyclonic (anticyclonic) axis, defined as the location where $\partial \zeta_{i}^{(0)} / \partial y=0$ and vertical vorticity is a local maximum (minimum). Terms II and III are of opposite sign but of different magnitudes such that momentum advection in the interior dominates the vorticity balance, causing cyclones to spin down faster than anticyclones. Nonlinear Ekman pumping at $O(\epsilon)$ tends to reduce this effect.

The $O(\epsilon)$ geostrophic current and the vertical relative vorticity solutions are

$$
\begin{aligned}
u_{i}^{(1)} & =\frac{13}{80} \mathrm{e}^{-t / 2} \frac{\partial}{\partial y} \int_{0}^{t} \mathrm{e}^{\tau / 2}\left(u_{i}^{(0)}\right)^{2} d \tau \\
\zeta_{i}^{(1)} & =\frac{13}{40} \mathrm{e}^{-t / 2} \int_{0}^{t} \mathrm{e}^{\tau / 2}\left(u_{i}^{(0)} \frac{\partial \zeta_{i}^{(0)}}{\partial y}-\left(\zeta_{i}^{(0)}\right)^{2}\right) d \tau .
\end{aligned}
$$

The nonlinear correction to the vertical relative vorticity shows that the total vertical relative vorticity is weakened on the cyclonic axis, where $\zeta_{i}^{(0)}>0$ and $\partial \zeta_{i}^{(0)} / \partial y=0$, and strengthened 


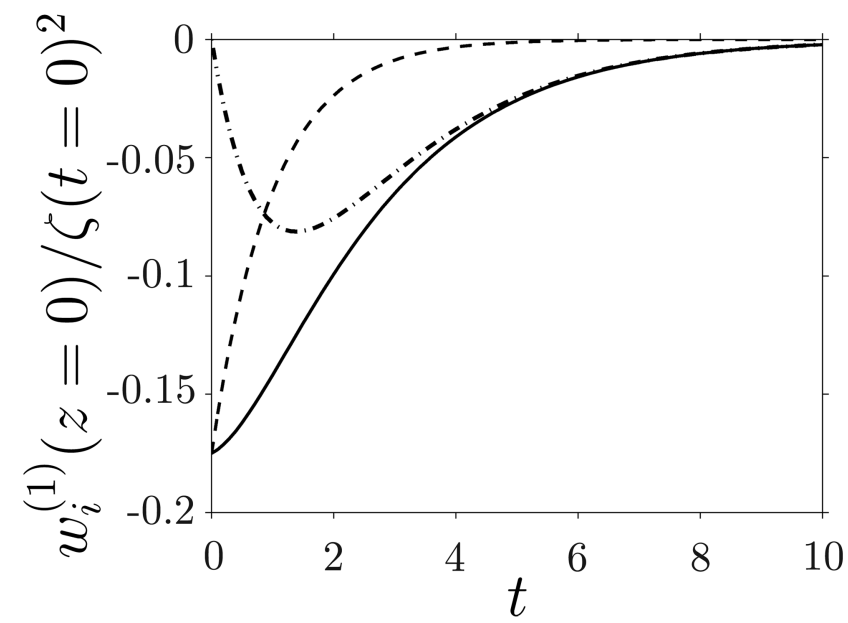

FIG. 2. The $O(\epsilon)$ Ekman pumping, which is scaled with the square of the initial relative vertical vorticity, is shown on the cyclonic or anticyclonic axis. The total correction (solid line) has contributions from Hart's ${ }^{3}$ formula (dashed line) as well as feedback with the interior geostrophic flow, which is predominantly modified by lateral momentum advection (dot-dashed line).

on the anticyclonic axis, where $\zeta_{i}^{(0)}<0$ and $\partial \zeta_{i}^{(0)} / \partial y=0$.

During spindown, the asymmetry in cyclonic vorticity, $\zeta_{c}$, and anticyclonic vorticity, $\zeta_{a}$, is measured by the ratio $\left|\zeta_{c} / \zeta_{a}\right|$. Consider the spindown of a cyclone and an anticyclone, where $\zeta_{c}(t=0)=-\zeta_{a}(t=0)$, of initial magnitude $|\zeta(t=0)|$. On the cyclonic and anticyclonic axis of the flow, the ratio of cyclonic to anticyclonic vorticity evolves as

$$
\begin{aligned}
\left|\frac{\zeta_{c}}{\zeta_{a}}\right| & =\frac{1-\epsilon F_{1}(t)}{1+\epsilon F_{1}(t)}, \text { where } \\
F_{1}(t) & =\frac{13}{20}|\zeta(t=0)|\left(1-\mathrm{e}^{-t / 2}\right) .
\end{aligned}
$$

At initial time, $F_{1}(t=0)=0$, but in the limit $t \rightarrow \infty, F_{1} \rightarrow(13 / 20)|\zeta(t=0)|$. Thus, the asymmetry in cyclonic and anticyclonic vorticity increases with time and increasing Rossby number.

Next, the complete solution to the time-dependent, $O(\epsilon)$ correction to the Ekman pumping solution is

$$
\begin{aligned}
w_{i}^{(1)}(z=0) & =\frac{7}{40}\left(u_{i}^{(0)} \frac{\partial \zeta_{i}^{(0)}}{\partial y}-\left(\zeta_{i}^{(0)}\right)^{2}\right) \\
& +\frac{13}{80} \mathrm{e}^{-t / 2} \int_{0}^{t} \mathrm{e}^{\tau / 2}\left(u_{i}^{(0)} \frac{\partial \zeta_{i}^{(0)}}{\partial y}-\left(\zeta_{i}^{(0)}\right)^{2}\right) d \tau
\end{aligned}
$$


Asymmetries in vertical vorticity and vertical velocity arising during nonlinear homogeneous spindown

On the cyclonic and anticyclonic axis, where $\partial \zeta_{i}^{(0)} / \partial y=0$, Ekman pumping becomes

$$
w_{i}^{(1)}(z=0)=-\frac{7}{40}\left(\zeta_{i}^{(0)}\right)^{2}-\frac{13}{40} \mathrm{e}^{-t / 2} \int_{0}^{t} \mathrm{e}^{\tau / 2}\left(\zeta_{i}^{(0)}\right)^{2} d \tau
$$

which is negative for all time. Thus, at $O(\epsilon)$, Ekman suction on the anticyclonic axis strengthens and Ekman pumping on the cyclonic axis weakens. The first term in the nonlinear correction, (32), is Hart's ${ }^{3}$ nonlinear Ekman pumping, which is nonzero at initial time. As shown in Figure 2, the second term in (32) provides a significant contribution to the asymmetry in Ekman pumping and suction due to feedback with the interior vorticity field. This time-dependent contribution is necessary for an accurate calculation of the $O(\epsilon)$ Ekman pumping and suction.

The asymmetry in Ekman pumping, $w_{p}$, and Ekman suction, $w_{s}$, is measured by the ratio $\left|w_{p} / w_{s}\right|$. Consider the time evolution of Ekman pumping and suction for cyclonic and anticyclonic vorticity, where $\zeta_{c}(t=0)=-\zeta_{a}(t=0)$ and $w_{p}(t=0)=-w_{s}(t=0)$, for vertical relative vorticity of initial magnitude $|\zeta(t=0)|$. On the cyclonic and anticyclonic axis of the flow, the ratio of Ekman pumping to Ekman suction evolves as

$$
\begin{aligned}
& \left|\frac{w_{p}}{w_{s}}\right|=\frac{1-\epsilon F_{2}(t)}{1+\epsilon F_{2}(t)}, \text { where } \\
& F_{2}(t)=\frac{13}{20}|\zeta(t=0)|\left(1-\frac{6}{13} \mathrm{e}^{-t / 2}\right) .
\end{aligned}
$$

At initial time, $F_{2}(t=0)=(7 / 20)|\zeta(t=0)|$, but in the limit $t \rightarrow \infty, F_{2} \rightarrow(13 / 20)|\zeta(t=0)|$. Within an inertial period, lateral Ekman advection of momentum leads to asymmetry in Ekman pumping and suction. With time, this asymmetry increases by momentum advection in the Ekman layer and the interior.

Figure 3 summarizes the roles of momentum advection in the Ekman layer and the interior in setting the asymmetry in Ekman pumping and suction and the asymmetric decay of cyclonic and anticyclonic vorticity.

\section{CONCLUSIONS}

During the nonlinear spindown of a geostrophic flow, two primary mechanisms lead to an asymmetry in Ekman pumping and suction as well as cyclonic and anticyclonic vorticity for increasing Rossby number. An analysis of the $O(\epsilon)$ correction shows that momentum advection in both the Ekman layer and the interior reduces Ekman pumping and enhances 

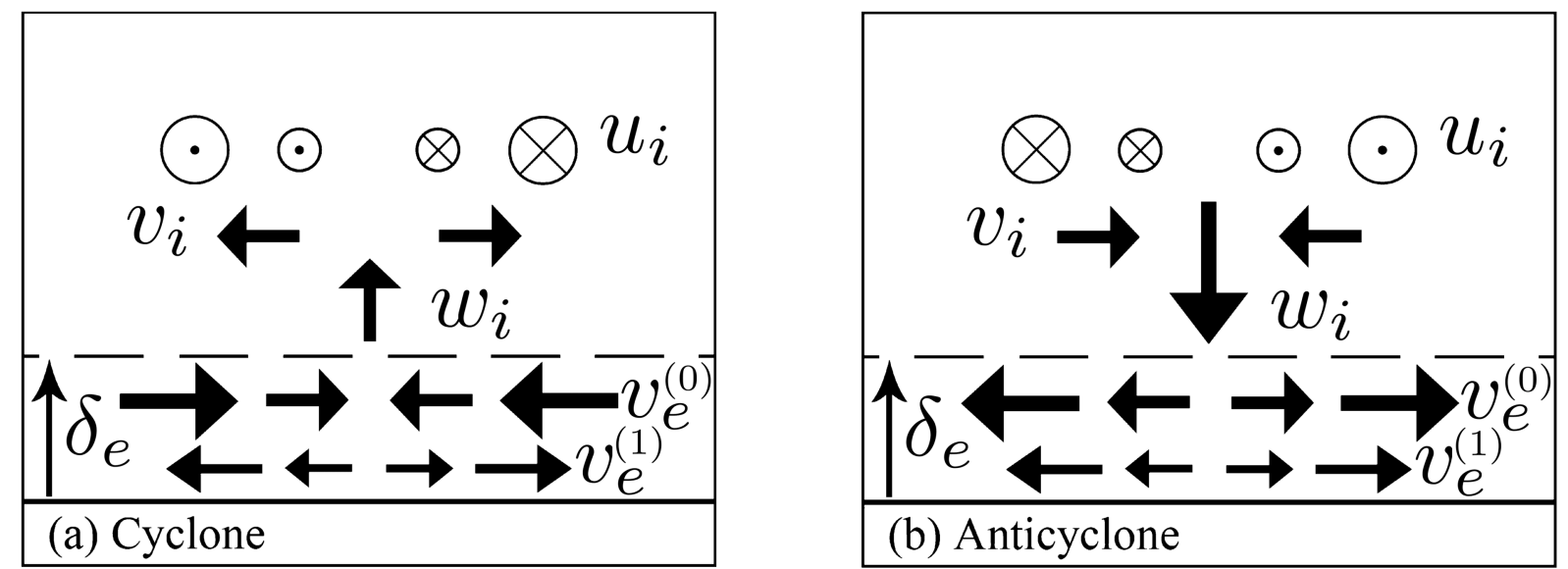

FIG. 3. Frictional spindown of a (a) cyclone and (b) anticyclone. A geostrophic flow, $u_{i}$, induces a leading order Ekman flow, $v_{e}^{(0)}$, that is directed laterally toward (away from) the cyclonic (anticyclonic) axis. Ekman advection of momentum contributes to an Ekman flow, $v_{e}^{(1)}$, that is directed laterally outward in both cases. This nonlinear correction weakens (strengthens) Ekman pumping, $w_{i}(z=0)$, from its zeroth-order approximation on the cyclonic (anticyclonic) axis. Ekman pumping drives an interior lateral circulation, $v_{i}$, that spins down the geostrophic flow. This secondary circulation also advects lower momentum fluid outward (inward), which causes the cyclone (anticyclone) to spin down faster (slower).

Ekman suction. The full time-dependent solution to $O(\epsilon)$ shows that horizontal momentum advection in the interior causes cyclonic vorticity to decay faster than anticyclonic vorticity despite weaker Ekman pumping than Ekman suction.

In the particular case of uniform vorticity, $\zeta(t)$, with unit amplitude at initial time, Hart $^{6}$ determines that the vertical relative vorticity's amplitude decays as

$$
|\zeta(t)|=\frac{1}{\mathrm{e}^{t / 2}\left(1+\gamma \epsilon \frac{13}{20}\right)-\gamma \epsilon \frac{13}{20}}
$$

Note that the above solution has been nondimensionalized as in Section II, and $\gamma=1$ (or -1) for cyclonic (anticyclonic) vorticity. By expanding this solution, the vertical relative vorticity to $O(\epsilon)$ is

$$
|\zeta(t)|=\mathrm{e}^{-t / 2}\left(1-\gamma \epsilon \frac{13}{20}\left(1-\mathrm{e}^{-t / 2}\right)\right)
$$

This solution is consistent with the general solution presented in Section III. For this case, a simple application of the $O(1)$ time-dependent spindown solution, (21), to (29) leads to 


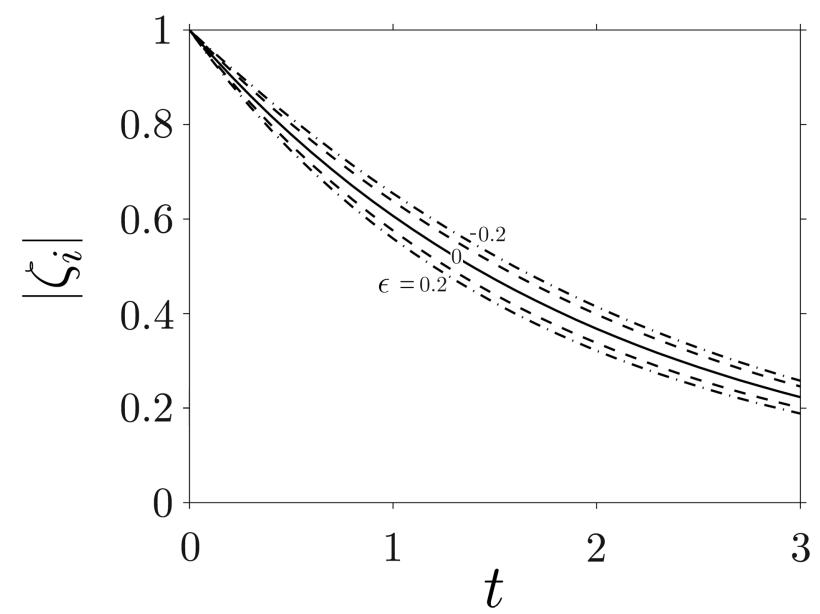

FIG. 4. The vertical relative vorticity is shown on the cyclonic or anticyclonic axis for the $O(1)$ solution (solid line) and to $O(\epsilon)$ with (dashed line) or without (dot-dashed line) the nonlinear Ekman pumping term. The solutions are shown for $|\epsilon|=0.2$, where $\epsilon>0$ is for cyclonic vorticity and $\epsilon<0$ is for anticyclonic vorticity.

the same expression and shows that the amplitude decays faster for cyclonic vorticity. This results supports Hart's ${ }^{6}$ conclusion that horizontal momentum advection in the interior dominates over the nonlinear correction to Ekman pumping during spindown.

The asymmetric spindown in vertical relative vorticity is also consistent with Zavala Sansón's ${ }^{8}$ findings in numerical experiments, in which the cyclonic vortices decay faster than anticyclonic vortices. Although the numerical simulations in that work neglect the nonlinear correction to Ekman pumping, the inclusion of the $O(\epsilon)$ correction in this work still shows the same behavior, a faster decay of cyclonic vorticity than anticyclonic vorticity. In order to evaluate how nonlinear Ekman pumping modifies the spindown solutions (29) and (31), new solutions are derived in which the nonlinear Ekman pumping term (the term in parentheses in (16)) is removed. Without this correction, the $O(\epsilon)$ solutions are

$$
\begin{aligned}
\zeta_{i}^{(1)} & =\frac{1}{2} \mathrm{e}^{-t / 2} \int_{0}^{t} \mathrm{e}^{\tau / 2}\left(u_{i}^{(0)} \frac{\partial \zeta_{i}^{(0)}}{\partial y}-\left(\zeta_{i}^{(0)}\right)^{2}\right) d \tau, \\
w_{i}^{(1)} & =\frac{1}{2} \zeta_{i}^{(1)}
\end{aligned}
$$

Figures 4 and 5 compare the spindown solutions to $O(\epsilon)$ with and without the nonlinear Ekman pumping term on the cyclonic or anticyclonic axis, where $|\zeta(t=0)|=1$, and $|\epsilon|=0.2$. Without the nonlinear Ekman pumping term, cyclonic (anticyclonic) vorticity 


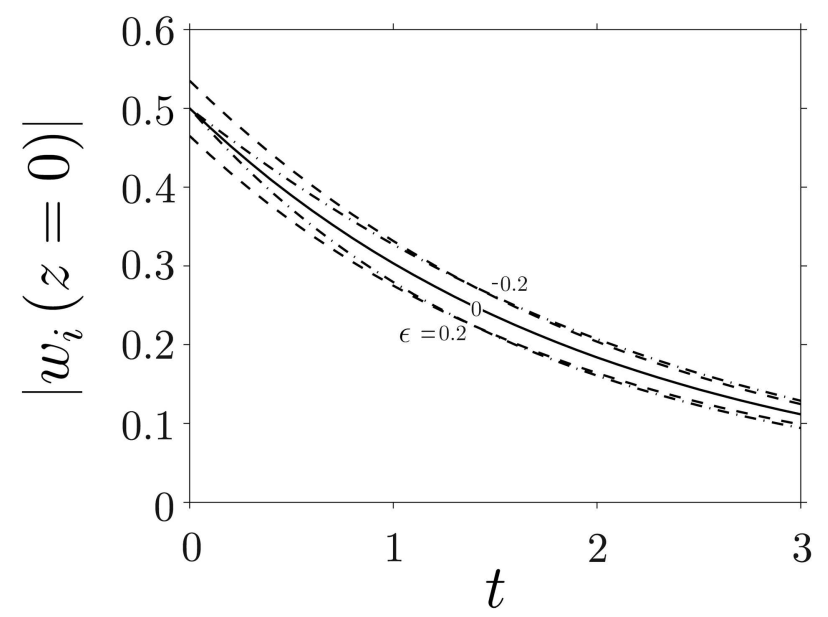

FIG. 5. The Ekman pumping solution is shown on the cyclonic or anticyclonic axis for the $O(1)$ solution (solid line) and with (dashed line) or without (dot-dashed line) the nonlinear correction to Ekman pumping.

appears to spin down more (less) rapidly. For the vertical velocity, neglect of this term initially overestimates (underestimates) Ekman pumping (suction) on the cyclonic (anticyclonic) axis. Interestingly, this solution reveals that the vertical velocity with or without this term becomes similar in magnitude after approximately a spindown time. The rapid weakening of the nonlinear Ekman pumping term's contribution to the total vertical velocity indicates that the linear Ekman pumping approximation may hold after a certain time, which supports previous studies ${ }^{7,8}$ use of linear Ekman pumping. However, without the nonlinear contribution to Ekman pumping, the measure of the vertical circulation within vortices will initially overestimate Ekman pumping and underestimate Ekman suction and lead to errors in the early spindown of vertical vorticity.

\section{ACKNOWLEDGMENTS}

J. A. Benthuysen was supported by the MIT/WHOI Joint Program. We also appreciate comments from two anonymous reviewers. 
Asymmetries in vertical vorticity and vertical velocity arising during nonlinear homogeneous spindown

\section{REFERENCES}

${ }^{1}$ J. G. Charney and A. Eliassen, "A numerical method of predicting the perturbations of the middle-latitude westerlies," Tellus 1, 38-54 (1949).

${ }^{2}$ G. S. Benton, F. B. Lipps, and S.-Y. Tuann, "The structure of the Ekman layer for geostrophic flows with lateral shear," Tellus XVI, 186-199 (1964).

${ }^{3}$ J. E. Hart, "A note on nonlinear corrections to the Ekman layer pumping velocity," Physics of Fluids 12, 131-135 (2000).

${ }^{4}$ K. H. Brink, "Time-dependent motions and the nonlinear bottom Ekman layer," Journal of Marine Research 55, 613-631 (1997).

${ }^{5}$ J. Pedlosky, "On the weakly nonlinear Ekman layer: Thickness and flux," Journal of Physical Oceanography 38, 1334-1339 (2008).

${ }^{6}$ J. E. Hart, "Nonlinear Ekman suction and ageostrophic effects in rapidly rotating flows," Geophysical and Astrophysical Fluid Dynamics 79, 201-222 (1995).

${ }^{7}$ L. Zavala Sansón and G. J. F. van Heijst, "Nonlinear Ekman effects in rotating barotropic flows," Journal of Fluid Mechanics 412, 75-91 (2000).

${ }^{8}$ L. Zavala Sansón, "The asymmetric Ekman decay of cyclonic and anticyclonic vortices," European Journal of Mechanics-B/Fluids 20, 541-556 (2001). 